\title{
The Minimum Leidenfrost Temperature on Smooth Surfaces
}

\author{
Dana Harvey, Joshua Méndez Harper, and Justin C. Burtor* \\ Department of Physics, Emory University, Atlanta, Georgia 30322, USA
}

(Dated: August 5, 2021)

\begin{abstract}
During the Leidenfrost effect, a thin insulating vapor layer separates an evaporating liquid from a hot solid. Here we demonstrate that Leidenfrost vapor layers can be sustained at much lower temperatures than those required for formation. Using a high-speed electrical technique to measure the thickness of water vapor layers over smooth, metallic surfaces, we find that the explosive failure point is nearly independent of material and fluid properties, suggesting a purely hydrodynamic mechanism determines this threshold. For water vapor layers of several millimeters in size, the minimum temperature for stability is $\approx 140^{\circ} \mathrm{C}$, corresponding to an average vapor layer thickness of $10-20 \mu \mathrm{m}$.
\end{abstract}

In his seminal 1756 treatise, J. G. Leidenfrost noted that a water droplet placed on a heated, polished metal spoon does not wet the surface [1. Instead, the water droplet levitates above the hot surface, cushioned by a vapor film generated by evaporation. Since then, the Leidenfrost effect has been well studied due to its importance in laboratory, industrial, and geophysical contexts [2]. Examples include the vapor layer geometry [3] 8 , spontaneous motion and oscillations of drops 9 15, drop impact on heated surfaces [16 19, and "nano-painting" through particle deposition [20, 21].

In nature, the Leidenfrost effect-or more precisely, the collapse of a Leidenfrost vapor layer between ascending magma and an aquifer-underpins one of the most energetic and common forms of volcanism: phreatomagmatic eruptions [22, 23. The Leidenfrost effect need not involve water or even a liquid; blocks of sublimating $\mathrm{CO}_{2}$ ice may "surf" down Martian dunes on lubricating layers of $\mathrm{CO}_{2}$ gas, carving channels and pits on the red planet's surface [24]. This very manifestation of the Leidenfrost effect may help power the first Martian colonies [25].

In all of these examples, precise knowledge of the transition temperature at which the vapor layer forms (or fails) is crucial. However, reported values of this temperature vary widely in the literature, and are known to depend on surface roughness [26 30 , hydrophobicity [29, 31 33, thermal properties of the solid [32 37, liquid temperature [29, 34, 35, 37 39], solid geometry 32 35, 37, 38, 40, 41, and liquid impurities [29, 36, 41, 42. For smooth, homogeneous surfaces, a comprehensive theoretical study by Zhao et al. 43 showed that the temperature at which the vapor layer forms spontaneously from a liquid-solid contact depends only on the hydrophobicity of the surface [43. For water drops on metallic surfaces, this corresponds to temperatures exceeding $200^{\circ} \mathrm{C}$. Yet, once formed, Leidenfrost drops can exist on metal surfaces with temperatures below boiling temperature $\left(100^{\circ} \mathrm{C}\right)$ 44.

Here, we show how this large metastable region between formation and failure arises from the hydrodynamic stability of the gas flow in the vapor layer. Our experiments employ a new electrical technique that can directly measure the average thickness of the vapor layer around a heated solid with microsecond resolution. For smooth metallic surfaces, we find a formation Leidenfrost temperature, $T_{+}$, consistent with recent predictions of a nanoscale wetting theory [43. Once a stable vapor layer is formed over a given solid surface, its thickness is solely a function of the surface temperature, $T_{s}$. Remarkably, we find a minimum Leidenfrost temperature, $T_{-}$, which is nearly independent of liquid impurities and solid properties. At this temperature, the vapor layer spontaneously fails through liquid-solid contact and rapid boiling.

Figure 17 illustrates how a small water droplet can levitate over a heated concave aluminum surface with temperature $T_{s}<T_{+}$. As the surface cools, the drop evaporates, but remains levitated (Video S1 45]). Eventually, liquid-solid contact occurs at $T_{-}$. This minimum temperature varies with drop size (Fig. 1b, Video S2 45]). Surprisingly, droplets smaller than $\approx 100 \mu \mathrm{m}$ can even levitate below the boiling point by way of a diffusive Stefan flow [46. Conversely, droplets with radii larger than the capillary length of water, $l_{c} \sim 2.5 \mathrm{~mm}$, fail at higher temperatures that are roughly independent of drop size. Large variations in $T_{-}$exist in this regime, possibly due to vapor layer oscillations sustained by evaporation [14, 47]. However, failure always occur near or below $T_{+}=190 \pm 20^{\circ} \mathrm{C}$, a value determined by carefully placing $\approx 2 \mathrm{~mm}$ drops of distilled water onto a heated, polished, concave aluminum surface until the drops did not break up or fizzle upon contact.

What determines $T_{-}$? Drops on flat surfaces with radii larger than $\approx 10 \mathrm{~mm}$ are known to succumb to the Rayleigh-Taylor instability [5, 6, yet Fig. 1b shows that all drops fail below a minimum temperature, regardless of size. To better investigate the thickness and dynamics of vapor layers with a well-controlled geometry, we used a heated metallic cylinder (diameter $=7.9 \mathrm{~mm}$ ) with a rounded tip immersed into a liquid bath heated to a temperature $T_{l}$ (Fig. 2a) so that a vapor layer forms around it. A ceramic heater and thermocouple were embedded in the cylinder, and the bath was heated externally. With this geometry, both $T_{s}$ and the water liquid level could be controlled independently. 

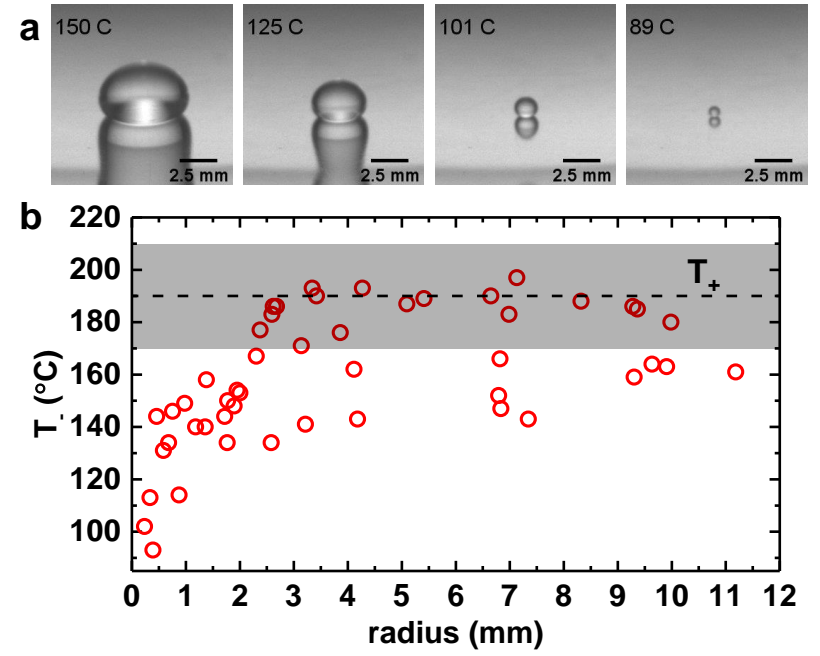

FIG. 1. (a) Sequence of images showing a single pure water Leidenfrost drop that remains levitated over a heated aluminum surface below the boiling point (Video S1 45]). (b) Failure temperature, $T_{-}$, as a function of drop radius. Each data point represents a different water drop. The upper Leidenfrost temperature, $T_{+}=190 \pm 20^{\circ} \mathrm{C}$, is indicated by the dashed line and shaded region.

To study the dynamics of the vapor layer at short time scales, we monitored the electrical impedance between the heated solid and a geometrically-similar lower electrode in the bath. The lower electrode was immersed completely in the liquid, whereas the heated electrode was immersed only to a depth of $H$ (see Fig. 2a). A 10 $\mathrm{MHz}$ signal was driven into the lower electrode and then measured at the heated electrode with a PC-based oscilloscope. The amplitude and phase of the signal were extracted by custom software-based lock-in detection (Fig. S1 [45]), as done in similar experiments investigating drop coalescence [49]. We added a variable concentration of $\mathrm{NaCl}$ salt to the bath in order to increase the conductivity of the liquid.

The region between the two electrodes, shown in the dashed box in Fig. 2a, can be modeled as an RLC circuit (Fig. 2b). The inductance, $L_{0}$, represents parasitic inductance in the experimental apparatus, and $R_{0}$ is the combined resistance of the liquid and metal-liquid boundaries. We treat the vapor layer as a capacitor, where one plate is the heated metal electrode and the other is the liquid surface (see Fig. 22). The interface between the upper, heated electrode and the liquid is modeled as a parallel combination of two capacitors. $C_{v}$ is the capacitance of the vapor layer, and $C_{\lambda}$ is the capacitance of the double layer that forms upon liquid-solid contact (Fig. S2 [45]). When the vapor layer is present, $C_{\lambda}=0$ and the average vapor layer thickness, $d$, can be computed using a simple geometric model. First, the vapor layer is modeled as a hemispherical capacitor with inner and a

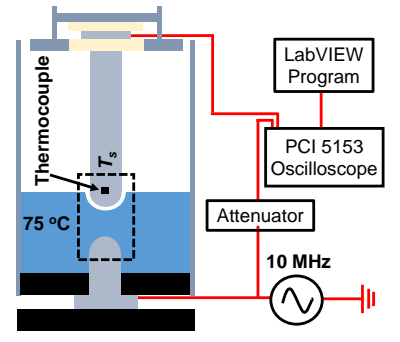

b
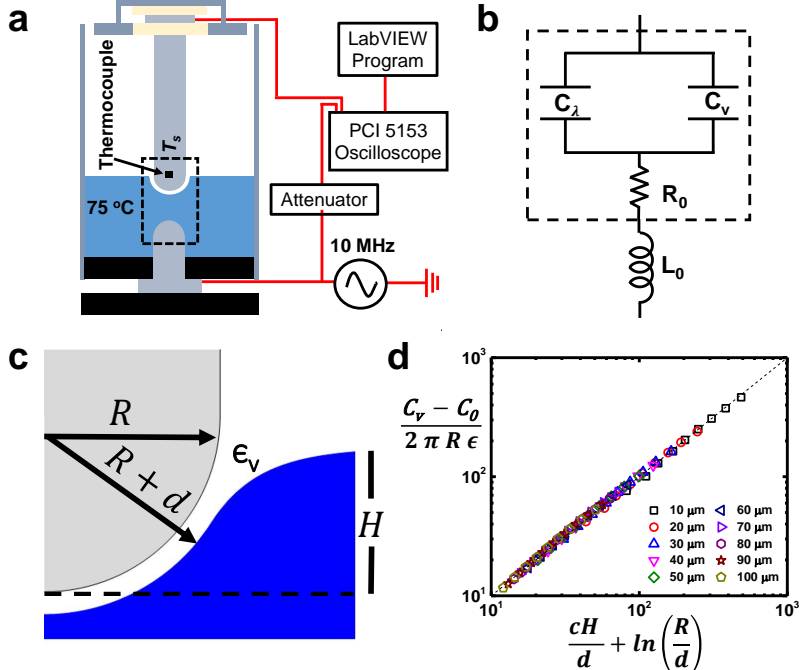

e

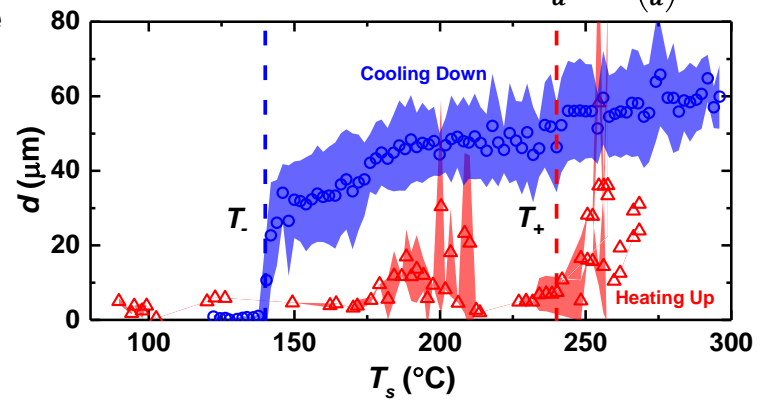

FIG. 2. (a) Experimental setup for high-speed measurements of the vapor layer dynamics. The dashed box indicates the complex impedance which varies in time. (b) Equivalent circuit for the complex impedance of the vapor layer, as described in the main text. Both $C_{v}$ and $C_{\lambda}$ are time dependent. (c) Simplified geometry for modeling the capacitance of the vapor layer. (d) Capacitance data from the COMSOL model [45, 48, using a range of values for $H$ and $d$. Symbols correspond to different values of $d$. The dashed line is Eq. 4 with $c=0.58$ and $C_{0}=1.85 \mathrm{pF}$. (e) $d$ versus $T_{s}$ for a nickel-coated copper electrode in pure water. The electrode was first heated (red triangles) and then subsequently cooled (blue circles). $T_{+}=240 \pm 30^{\circ} \mathrm{C}$ (red dashed line) was the average temperature when the vapor layer formed, and $T_{-}=140 \pm 10^{\circ} \mathrm{C}$ (blue dashed line) was the average temperature when the vapor layer collapsed. The shaded regions show the standard deviation from multiple experiments.

outer radii equal to $R$ and $R+d$, respectively:

$$
C_{1}=2 \pi \epsilon_{v} \frac{R(R+d)}{d} \times c \frac{H}{R} .
$$

The first term corresponds to a hemispherical capacitor. However, as shown in Fig. 22, the vapor layer does not encompass the entire volume between the two hemispheres for a given value of the immersion depth $H$. To lowest order, we modified the volume of the vapor layer by a factor of $c H / R$, where $c$ is a numerical constant that takes into account the curved water surface away from the electrode. 
In our experiments, $R=7.9 \mathrm{~mm}$ and $\epsilon_{v}=1.0057 \epsilon_{0}$ is the dielectric constant of water vapor at $100^{\circ} \mathrm{C}$. Since $d / R<1.5 \%$, we only consider the leading order term so that

$$
C_{1}=2 \pi c H \epsilon_{v} \frac{R}{d}
$$

As the water level is lowered $(H \rightarrow 0)$, the capacitance approaches that of a sphere above a flat plane. To leading order, this is given by

$$
C_{2}=2 \pi \epsilon_{v} R \ln \left(\frac{R}{d}\right)+C_{0}
$$

$C_{0}$ is constant that contains the residual capacitance of the rest of the cylindrical electrode. Combing these terms gives us a relationship between $C_{v}=C_{1}+C_{2}$ and $d$ :

$$
\frac{C_{v}-C_{0}}{2 \pi R \epsilon_{v}}=\frac{c H}{d}+\ln \left(\frac{R}{d}\right) .
$$

To verify Eq. 4, we generated the equivalent electrostatic geometry in COMSOL and measured the resulting capacitance, as described in the supplemental material [45, 48, The water surface profile was computed by a surface of revolution composed of two curves: 1) an arc of a circle with radius $R+d$ and 2) a hydrostatic solution of the water's surface profile taking surface tension and gravity into account [50]. The two curves were matched at a point with continuous first derivatives, providing a unique solution given the boundary conditions (Fig. S3 [45]). By simulating many curves with $1.3 \mathrm{~mm}<H<$ $8.3 \mathrm{~mm}$ and $10 \mu \mathrm{m}<d<100 \mu \mathrm{m}$, we found excellent agreement with Eq. 4, as shown in Fig. 2 $\mathrm{d}$. The data collapses well for $c=0.58$ and $C_{0}=1.85 \mathrm{pF}$. To find $d$ from measurements of $C_{v}$, Eq. 4 was analytically solved in terms of product logarithms.

Upon heating and then cooling the immersed upper electrode, we observed a large, metastable region characterized by hysteresis in $d$ versus $T_{s}$, in agreement with Fig. 1 b. Figure 2 s shows the average vapor layer thickness between pure water at $T_{l}=75-95^{\circ} \mathrm{C}$ and a nickelcoated copper electrode. For this particular experiment, $\mathrm{NaCl}$ was not added to avoid salt deposition on the electrode surface. Bubble nucleation and detachment resulted in large variations in our measurements of $d$ during heating. On average, a stable vapor layer formed at $T_{+}=240 \pm 30^{\circ} \mathrm{C}$, which is consistent with recent theoretical predictions for metallic surfaces [43. Nevertheless, once formed, the vapor layer remained stable at temperatures well below $T_{+}$. As shown in Fig. 2e, the collapse of the vapor layer occurred repeatedly at $T_{-}=140 \pm 10^{\circ} \mathrm{C}$. Video S3 [45] shows a time lapse of a characteristic experiment.

The collapse of the vapor layer at $T_{-}$is explosive and audible. We used synchronized high-speed video (Phantom V7.11, Vision Research) to visualize this process.

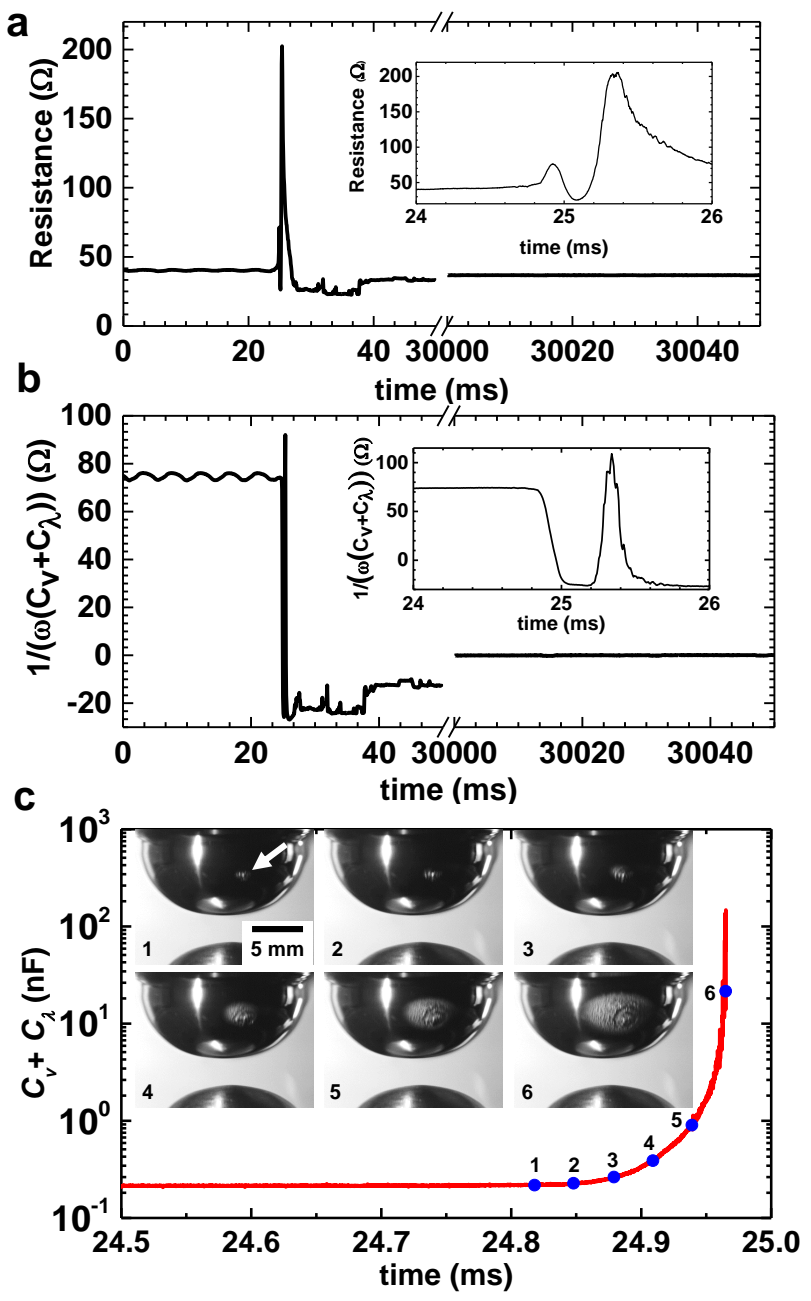

FIG. 3. Resistance (a) and capacitive reactance (b) of the Leidenfrost cell during collapse with $0.02 \mathrm{M} \mathrm{NaCl}$. The insets show $2 \mathrm{~ms}$ of the data right before and after collapse. $30 \mathrm{~s}$ after collapse, the system is quiescent. Capillary waves are visible as oscillations in the reactance prior to collapse. (c) Total capacitance of the liquid-vapor-solid interface just before collapse. The enormous increase is due to the formation of an ionic double layer at the liquid-solid contact. The images show a time sequence of the initial collapse point, as indicated by the arrow, where bubbles are generated as the wetting front spreads rapidly. The blue points in the data correspond to the indicated images (Video S4).

Figure $3 \mathrm{a}$ and $3 \mathrm{~b}$ show the real and imaginary parts of the impedance (dashed box in Fig. 2 $\mathrm{p}$ ) before and after a single collapse event. The bubbles generated during the explosion lead to a large increase in resistance before eventually returning to quiescence at long times. The slightly smaller resistance after collapse was due to the increased water temperature from the heated electrode. For some values of $H$, we observed capillary waves that traveled upwards along the vapor layer. These manifested as oscillations in the reactive impedance (Fig. $3 \mathrm{~b}$ ). 
In Video S4 45, the capillary waves are visible with a typical wavelength of $\lambda=2-3 \mathrm{~mm}$. We can estimate the corresponding frequency using the dispersion relation for pure capillary waves, $f=\left(\gamma k^{3} / \rho_{l}\right)^{1 / 2} / 2 \pi \approx 120-220 \mathrm{~Hz}$, where $\rho_{l}=959 \mathrm{~kg} / \mathrm{m}^{3}$ is the density of pure water at the boiling point, and $k=2 \pi / \lambda$ is the wave vector. This agrees well with Fig. 3 b, where $f \approx 200 \mathrm{~Hz}$.

During collapse, the combined capacitance, $C_{v}+C_{\lambda}$, increases by more than 3 orders of magnitude (Fig. 3c), and is facilitated by an explosive wetting front spreading from the initial contact point. The speed of this front is consistent with the capillary velocity, $\gamma / \eta_{w} \approx 210 \mathrm{~m} / \mathrm{s}$, where $\gamma=59 \mathrm{mN} / \mathrm{m}$ is the liquid-vapor surface tension and $\eta_{w}=0.28 \mathrm{mPa} \cdot \mathrm{s}$ is the viscosity of water at the boiling point. The large increase in capacitance is due to the formation of an ionic double layer as soon as the liquid contacts the surface, made possible by the addition of salt in the water (Fig. S2 [45]). The effective thickness of the ionic screening layer (1-10 nm [51]) is 3 orders of magnitude smaller than the thickness of the vapor layer, resulting in a much larger capacitance. Thus, even a small fraction of liquid touching the electrode surface will drastically increase the capacitance. The slower decay in the impedance is due to the dissipation of a large cloud of vapor bubbles (Video S4 [45]).

Surprisingly, and in contrast to the strong dependence of $T_{+}$on material properties [43, we found that $T_{-}$was independent of the metal used for the heated electrode. Figure 4 a shows $d$ versus $T_{s}$ for 3 representative experiments with metals of varying thermal conductivity: tita$\operatorname{nium}(7 \mathrm{~W} / \mathrm{m} \cdot \mathrm{K})$, brass $(115 \mathrm{~W} / \mathrm{m} \cdot \mathrm{K})$, and copper $(390$ $\mathrm{W} / \mathrm{m} \cdot \mathrm{K})$. For each material, the time evolution of $T_{s}$ looked distinct due to differences in heat capacity (Fig. S4 [45]), yet $d$ only depended on $T_{s}$. The discontinuities in the data at lower temperatures mark the failure of the vapor layer and determine both $T_{-}$and the spatiallyaveraged vapor layer thickness at collapse, $d_{c}$. These values were independently measured in each experiment. Figure $4 \mathrm{~b}$ shows $T_{-}$and $d_{c}$ for different metals, liquid levels $H$, and aqueous salt concentrations. A larger thermal conductivity resulted in slightly larger values of $d_{c}$. We speculate that localized cooling near the liquid interface [52] could result in a smaller vapor layer thickness near the tip of the electrode for metals with lower thermal conductivity. However, the stability of the vapor pocket should be mostly determined by the gas flux through the "neck" region [6], where vapor layer opens up to ambient pressure.

Furthermore, both $T_{-}$and $d_{c}$ were nearly independent over the range $3.7 \mathrm{~mm}<H<7.6 \mathrm{~mm}$ (Fig. S5), which is consistent with the behavior of Leidenfrost drops shown in Fig. 1 $\mathrm{b}$. This range of $H$ corresponded to vapor layer surface areas of $89-210 \mathrm{~mm}^{2}$, as computed from the geometric model (Fig. S3). Although we did not investigate metal geometries with $R \lesssim l_{c}$, we would expect a significant drop in $T_{-}$in this regime due to a lack of vapor layer
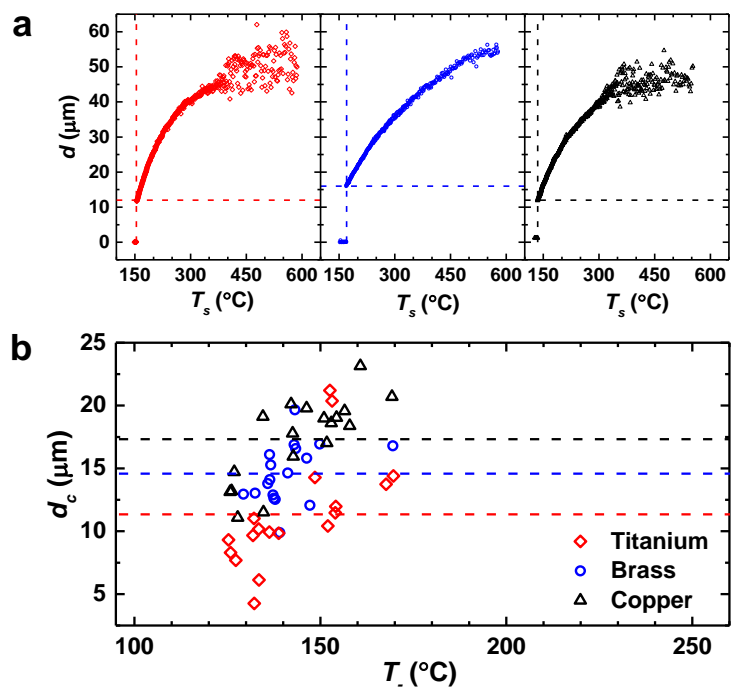

FIG. 4. (a) Spatially-averaged vapor layer thickness, $d$, as a function of substrate temperature, $T_{s}$, during the cooling of titanium, brass, and copper electrodes. The visible discontinuities in the data, indicated by the dashed lines, correspond to vapor layer failure at temperature $T_{-}$and thickness $d_{c}$ (b) Thickness at failure, $d_{c}$, as a function of the temperature at failure, $T_{-}$. Each data point represents varying aqueous $\mathrm{NaCl}$ concentrations and liquid level $H$ for each metal. The dashed lines show the mean value of $d_{c}$ for each metal, while the average of $T_{-}$was $140 \pm 10^{\circ} \mathrm{C}$ for all experiments.

fluctuations [3, 47. Additionally, $T_{-}$and $d_{c}$ showed no dependence on $\mathrm{NaCl}$ salt concentration (Fig. S6a). The addition of salt is widely known to suppress Leidenfrost phenomena [36, 41, 42, despite the fact that $\mathrm{NaCl}$ concentrations even up to sea water do not strongly affect the vapor pressure [53], evaporation rate, boiling point, viscosity [54], or surface tension of water [55]. Taken together, these measurements suggest that the minimum Leidenfrost temperature is determined by the hydrodynamic stability of the vapor layer. For water, failure reliably occurs at $T_{-}=140 \pm 10^{\circ} \mathrm{C}$ and $d_{c} \approx 10-20 \mu \mathrm{m}$.

Although Leidenfrost vapor layers require a materialdependent elevated temperature $\left(T_{+}\right)$for formation, here we showed how vapor layers can be stable at a much lower temperature $\left(T_{-}\right)$that is nearly independent of material and liquid properties. These two temperatures can be separated by more than $100^{\circ} \mathrm{C}$, leading to a large hysteresis and an explosive collapse at low temperatures. This study inherently poses outstanding questions surrounding the initiation of vapor layer collapse, either through unsteady hydrodynamic fluctuations or surface roughness. The liquid interface must approach the surface on sub-micron length scales for short-ranged Van der Waals forces to initiate contact and wetting [43. We suspect that in highly-dynamic geometries where the vapor layer is constantly in motion, hysteresis may not be visible due to repeated liquid-solid contacts 30. Nevertheless, this 
study explains the surprising robustness of Leidenfrost vapor layers once they are formed, and the physics that determines their violent demise.

This work was supported by the NSF DMR Grant No. 1455086 .

* justin.c.burton@emory.edu

[1] J. G. Leidenfrost, De aquae communis nonnullis qualitatibus tractatus (Ovenius, 1756).

[2] D. Quéré, Leidenfrost dynamics, Annu. Rev. Fluid Mech. 45, 197 (2013).

[3] J. C. Burton, A. L. Sharpe, R. C. A. van der Veen, A. Franco, and S. R. Nagel, Geometry of the vapor layer under a leidenfrost drop, Phys. Rev. Lett. 109, 074301 (2012).

[4] S. B., A. Rednikov, S. Dorbolo, and P. Colinet, Leidenfrost effect: Accurate drop shape modeling and refined scaling laws, Phys. Rev. E 90, 053011 (2014).

[5] A.-L. Biance, C. Clanet, and D. Quéré, Leidenfrost drops, Phys. Fluids 15, 1632 (2003).

[6] J. H. Snoeijer, P. Brunet, and J. Eggers, Maximum size of drops levitated by an air cushion, Phys. Rev. E 79, 053011 (036307).

[7] L. Duchemin, J. R. Lister, and U. Lange, Static shapes of levitated viscous drops, J. Fluid Mech. 533, 161 (2005).

[8] J. R. Lister, A. B. Thompson, A. Perriot, and L. Duchemin, Shape and stability of axisymmetric levitated viscous drops, J. Fluid Mech. 617, 167 (2008).

[9] A. Bouillant, T. Mouterde, P. Bourrianne, A. Lagarde, C. Clanet, and D. Quéré, Leidenfrost wheels, Nature Physics (2018).

[10] T. R. Cousins, R. E. Goldstein, J. W. Jaworski, and A. I. Pesci, A ratchet trap for Leidenfrost drops, J. Fluid Mech. 696, 215 (2012).

[11] G. Lagubeau, M. Le Merrer, C. Clanet, and D. Quéré, Leidenfrost on a ratchet, Nature Phys. 7, 395 (2011).

[12] H. Linke, B. J. Alemán, L. D. Melling, M. J. Taormina, M. J. Francis, C. C. Dow-Hygelund, V. Narayanan, R. P. Taylor, and A. Stout, Self-propelled Leidenfrost droplets, Phys. Rev. Lett. 96, 154502 (2006).

[13] A. Gauthier, C. Diddens, R. Proville, D. Lohse, and D. van der Meer, Self-propulsion of inverse leidenfrost drops on a cryogenic bath, Proc. Natl. Acad. Sci. USA 116, 1174 (2019).

[14] X. Ma and J. C. Burton, Self-organized oscillations of leidenfrost drops, Journal of Fluid Mechanics 846, 263 (2018).

[15] X. Ma, J. Liétor-Santos, and J. C. Burton, Star-shaped oscillations of leidenfrost drops, Physical Review Fluids 2, 031602(R) (2017).

[16] T. Tran, H. J. J. Staat, A. Prosperetti, C. Sun, and D. Lohse, Drop impact on superheated surfaces, Phys. Rev. Lett. 108, 036101 (2012).

[17] G. Castanet, O. Caballina, and F. Lemoine, Drop spreading at the impact in the leidenfrost boiling, Phys. Fluids 27, 063302 (2015).

[18] G. Riboux and J. M. Gordillo, Maximum drop radius and critical weber number for splashing in the dynamical leidenfrost regime, J. Fluid Mech. 803, 516-527 (2016)

[19] S.-C. Yao and K. Y. Cai, The dynamics and leidenfrost temperature of drops impacting on a hot surface at small angles, Exp. Therm. and Fluid Sci. 1, 363 (1988).

[20] C. Bain, Drip painting on a hot canvas, Nature Nanotechnology 2, 344 (2007).

[21] M. Elbahri, D. Paretkar, K. Hirmas, S. Jebril, and R. Adelung, Anti-lotus effect for nanostructuring at the leidenfrost temperature, Advanced Materials 19, 1262 (2007).

[22] V. Lorenz, Maar-diatreme volcanoes, their formation, and their setting in hard-rock or soft-rock environments, Geolines 15, 72 (2003).

[23] L. De León-Barragán, G. Carrasco-Núñez, and M. H. Ort, Stratigraphy and evolution of the holocene aljojuca maar volcano (serdán-oriental basin, eastern transmexican volcanic belt), and implications for hazard assessment, Journal of Volcanology and Geothermal Research 392, 106789 (2020).

[24] L. Mc Keown, M. Bourke, and J. McElwaine, Experiments on sublimating carbon dioxide ice and implications for contemporary surface processes on mars, Scientific reports 7, 1 (2017).

[25] G. G. Wells, R. Ledesma-Aguilar, G. McHale, and K. Sefiane, A sublimation heat engine, Nature communications 6, 1 (2015).

[26] H. Kim, B. Truong, J. Buongiorno, and L.-W. Hu, On the effect of surface roughness height, wettability, and nanoporosity on leidenfrost phenomena, Appl. Phys. Lett. 98, 083121 (2011).

[27] S. H. Kim, H. S. Ahn, J. Kim, M. Kaviany, and M. H. Kim, Dynamics of water droplet on a heated nanotubes surface, Appl. Phys. Lett. 102, 233901 (2013).

[28] C. Kruse, T. Anderson, C. Wilson, C. Zuhlke, D. Alexander, G. Gogos, and S. Ndao, Extraordinary shifts of the leidenfrost temperature from multiscale micro/nanostructured surfaces, Langmuir 29, 9798 (2013).

[29] H. Kim, J. Buongiorno, L. Hu, and T. Mckrell, Nanoparticle deposition effects on the minimum heat flux point and quench front speed during quenching in water-based alumina nanofluids, International Journal of Heat and Mass Transfer 53, 1542 (2010).

[30] P. R. Jones, C. Chuang, T. Sun, T. Y. Zhao, K. Fezzaa, J. C. Takase, D. Singh, and N. A. Patankar, High-speed x-ray imaging of the leidenfrost collapse, Scientific Reports 9, 1598 (2019).

[31] G. Liu and V. S. J. Craig, Macroscopically flat and smooth superhydrophobic surfaces: Heating induced wetting transitions up to the leidenfrost temperature, Faraday Discussions 146, 141 (2010).

[32] I. U. Vakarelski, J. O. Marston, D. Y. C. Chan, and S. T. Thoroddsen, Drag reduction by leidenfrost vapor layers, PRL 106, 214501 (2011).

[33] I. Vakarelski, N. A. Patankar, J. O. Marston, D. Y. C. Chan, and S. T. Thoroddsen, Stabilization of liedenfrost vapour layer by textured superhydrophobic surfaces, Nature 489, 274 (2012).

[34] R. Freud, R. Harari, and E. Sher, Collapsing criteria for vapor film around solid spheres as a fundamental stage leading to vapor explosion, Nuclear Engineering and Design 239, 722 (2009).

[35] V. V. Yagov, A. R. Zabirov, and M. A. Lexin, Unsteady heat transfer during subcooled film boiling, Heat and Mass Transfer and Properties of Working Fluids and Materials 62, 833 (2015).

[36] S. Hsu, Y. Ho, M. Ho, J. Wang, and C. Pan, On the 
formation of vapor film during quenching in de-ionized water and elimination of film boiling during quenching in natural sea water, International Journal of Heat and Mass Transfer 86, 65 (2015).

[37] I. Sher, R. Harari, R. Reshef, and E. Sher, Film boiling collapse in solid spheres immersed in a sub-cooled liquid, Applied Thermal Engineering 36, 219 (2012).

[38] H. Jouhara and B. P. Axcell, Film boiling heat transfer and vapour film collapse on spheres, cylinders, and plane surfaces, Nuclear Engineering and Design 239, 1885 (2009).

[39] V. V. Yagov, M. A. Lexin, A. R. Zabirov, and O. N. Kaban'kov, Film boiling of subcooled liquids. part i: Leidenfrost phenomenon and experimental results for subcooled water, International Journal of Heat and Mass Transfer 100, 908 (2016).

[40] W. S. Bradfield, Liquid-solid contact in stable film boiling, Ind. Eng. Chem. Fundamen. 5, 200 (1966).

[41] C.-K. Huang and V. P. Carey, The effects of dissolved salt on the leidenfrost transition, International Journal of Heat and Mass Transfer 50, 269 (2007).

[42] K. H. M. Abdalrahman, Sabariman, and E. Specht, Influence of salt mixture on the heat transfer during spray cooling of hot metals, International Journal of Heat and Mass Transfer 78, 76 (2014).

[43] T. Y. Zhao and N. A. Patankar, The thermo-wetting instability driving leidenfrost film collapse, PNAS 117, 13321 (2020).

[44] K. J. Baumeister, R. C. Hendricks, and T. D. Hamill, Metastable leidenfrost states, NASA Technical Note D3226 (1966).

[45] See Supplemental Material at [URL will be inserted by publisher] for videos and a detailed description of the experiments and simulations.
[46] D. V. Zaitsev, D. P. Kirichenko, V. S. Ajaev, and O. A. Kabov, Levitation and self-organization of liquid microdroplets over dry heated substrates, Phys. Rev. Lett. 119, 094503 (2017).

[47] T. A. Caswell, Dynamics of the vapor layer below a leidenfrost drop, Physiscal Review E 90, 013014 (2014).

[48] COMSOL Multiphysicsß, v. 5.3. COMSOL AB, Stockholm, Sweden (2020).

[49] J. D. Paulsen, J. C. Burton, and S. R. Nagel, Viscous to inertial crossover in liquid drop coalescence, PRL 106, 114501 (2011).

[50] J. C. Burtin, F. M. Huisman, P. Alison, D. Rogerson, and P. Taborek, Experimental and numerical investigation of the equilibrium geometry of liquid lenses, Langmuir Article 26, 15316-15324 (2010).

[51] M. Khademi and D. P. J. Barz, Structure of the electrical double layer revisited: Electrode capacitance in aqueous solutions, Langmuir 36, 4250 (2020).

[52] M. A. J. van Limbeek, M. H. Klein Schaarsberg, B. Sobac, A. Rednikov, C. Sun, P. Colinet, and D. Lohse, Leidenfrost drops cooling surfaces: theory and interferometric measurement, J. Fluid Mech. 827, 614 (2017).

[53] B. M. Fabuss and A. Korosi, Vapor pressures of binary aqueous solutions of nacl, $\mathrm{kcl}, \mathrm{na}_{2} \mathrm{SO}_{4}$, and $\mathrm{mgsO}_{4}$ at concentrations of interest in desalination processes, Desalination 1, 139 (1966).

[54] J. Kestin, H. E. Khalifa, and C. R. J., Tables of the dynamic and kinematic viscosity of aqueous nacl solutions in the temperature range $20-150^{\circ} \mathrm{c}$ and the pressure range 0.1-35 mpa, Journal of Physical and Chemical Reference Data 10, 71 (1981).

[55] G. Jones and W. A. Ray, The surface tension of solutions of electrolytes as a function of the concentration. iii. sodium chloride, J. Am. Chem. Soc 63, 3262 (1941). 